\title{
Sm protein methylation is dispensable for snRNP assembly in Drosophila melanogaster
}

\author{
GRAYDON B. GONSALVEZ, ${ }^{1,2}$ KAVITA PRAVEEN, ${ }^{1,2}$ AMANDA J. HICKS, ${ }^{1,2}$ LIPING TIAN, $^{2}$ \\ and A. GREGORY MATERA ${ }^{1,2}$ \\ ${ }^{1}$ Department of Biology, Program in Molecular Biology and Biotechnology, Lineberger Comprehensive Cancer Center, University of \\ North Carolina at Chapel Hill, Chapel Hill, North Carolina 27599-3280, USA \\ ${ }^{2}$ Department of Genetics, School of Medicine, Case Western Reserve University, Cleveland, Ohio 44106-4955, USA
}

\begin{abstract}
Sm proteins form stable ribonucleoprotein (RNP) complexes with small nuclear (sn)RNAs and are core components of the eukaryotic spliceosome. In vivo, the assembly of $\mathrm{Sm}$ proteins onto snRNAs requires the survival motor neurons (SMN) complex. Several reports have shown that SMN protein binds with high affinity to symmetric dimethylarginine (sDMA) residues present on the C-terminal tails of SmB, SmD1, and SmD3. This post-translational modification is thought to play a crucial role in snRNP assembly. In human cells, two distinct protein arginine methyltransferases (PRMT5 and PRMT7) are required for snRNP biogenesis. However, in Drosophila, loss of Dart5 (the fruit fly PRMT5 ortholog) has little effect on snRNP assembly, and homozygous mutants are completely viable. To resolve these apparent differences, we examined this topic in detail and found that Drosophila Sm proteins are also methylated by two methyltransferases, Dart5/PRMT5 and Dart7/PRMT7. Unlike dart5, we found that dart7 is an essential gene. However, the lethality associated with loss of Dart7 protein is apparently unrelated to defects in snRNP assembly. To conclusively test the requirement for sDMA modification of Sm proteins in Drosophila snRNP assembly, we constructed a fly strain that exclusively expresses an isoform of SmD1 that cannot be sDMA modified. Interestingly, these flies were viable, and snRNP assays revealed no defects in comparison to wild type. In contrast, dart5 mutants displayed a strong synthetic lethal phenotype in the presence of a hypomorphic Smn mutation. We therefore conclude that dart5 is required for viability when SMN is limiting.
\end{abstract}

Keywords: snRNP biogenesis; Dart5; Dart7; PRMT5; PRMT7; arginine methyltransferase; SMN; SMA

\section{INTRODUCTION}

Small ribonucleoproteins (RNPs) are highly abundant in eukaryotic cells and are involved in a diverse array of processes, from ribosome maturation to telomere maintenance (for review, see Matera et al. 2007). Pre-messenger RNA splicing is carried out by snRNPs of the Sm and Sm-like (Lsm) classes. The U1, U2, U4, and U5 snRNPs contain a common set of seven core Sm proteins, whereas the U6 snRNP contains a related set of Lsm proteins. With the exception of U6, which is thought to assemble exclusively in the nucleus, maturation of the other spliceosomal snRNPs involves both nuclear and cytoplasmic phases (Matera et al. 2007).

Reprint requests to: A. Gregory Matera, Department of Biology, Program in Molecular Biology and Biotechnology, Lineberger Comprehensive Cancer Center, University of North Carolina, 415 Fordham Hall, CB 3280, Chapel Hill, NC 27599-3280, USA; e-mail: agmatera@email.unc.edu; fax: (919) 962-8472.

Article published online ahead of print. Article and publication date are at http://www.rnajournal.org/cgi/doi/10.1261/rna.940708.
The life cycle of Sm-class snRNPs begins in the nucleus, where the snRNA is transcribed by RNA polymerase II and subsequently is transported to the cytoplasm via the export factors PHAX and CRM1 (Ohno et al. 2000). Once in the cytoplasm, Sm proteins are assembled onto the snRNA by the survival motor neurons (SMN) complex (for reviews, see Meister et al. 2002; Paushkin et al. 2002). Next, the 7methylguanosine cap of the snRNA is hypermethylated to a 2,2,7-trimethylguanosine (TMG) cap by the enzyme TGS1 (Mouaikel et al. 2002, 2003). The TMG cap serves as a docking site for the import adaptor Snurportin1 (Huber et al. 1998). Together with the import receptor Importin- $\beta$, Snurportin1 and the SMN complex mediate import of the snRNP into the nucleus (Massenet et al. 2002; Narayanan et al. 2002, 2004; Ospina et al. 2005). Additional rounds of modification and assembly are thought to take place in Cajal bodies (Jady et al. 2003; Nesic et al. 2004; Matera et al. 2007) before the snRNP is finally ready to function in premRNA splicing.

The cytoplasmic phase of snRNP assembly is particularly interesting from a medical standpoint, because mutations 
that reduce SMN protein levels result in the inherited neuromuscular disorder, spinal muscular atrophy (SMA) (Lefebvre et al. 1995). Current animal models implicate snRNP-dependent and -independent functions of SMN as etiological factors in SMA (Winkler et al. 2005; Carrel et al. 2006; Avila et al. 2007; Gabanella et al. 2007; Rajendra et al. 2007). Thus, it is imperative to understand in detail the mechanism by which SMN recognizes Sm proteins and snRNAs to facilitate efficient snRNP assembly. In metazoa, three of the seven $\mathrm{Sm}$ proteins $(\mathrm{SmB}, \mathrm{SmD1}$, and $\mathrm{SmD} 3$ ) are post-translationally modified to contain symmetric dimethylarginine (sDMA) residues within their C-terminal tails (Brahms et al. 2000, 2001). This modification is catalyzed by protein arginine methyltransferases (PRMTs) of the type II category. Enzymes of the type I category catalyze the more common asymmetric dimethylarginine (aDMA) modification (for review, see Bedford and Richard 2005). We recently demonstrated that, in human cells, sDMA modification of Sm proteins is carried out by two distinct type II methyltransferases, PRMT5 and PRMT7 (Gonsalvez et al. 2007). Furthermore, consistent with previous inhibitor-based studies (Brahms et al. 2001; Friesen et al. 2001), depletion of either enzyme interfered with the ability of SMN to efficiently recognize the Sm proteins (Gonsalvez et al. 2007). Consequently, cytoplasmic snRNP assembly was disrupted by depletion of either PRMT5 or PRMT7. Thus, sDMA modification of Sm proteins serves an important regulatory function in human cells.

In contrast, the situation observed in Drosophila is quite different. Loss of Dart5/Capsuléen (CG3730; the fly PRMT5 ortholog) activity results in production of Sm proteins that are not recognized by two sDMA-specific antibodies, Y12 and SYM10 (Gonsalvez et al. 2006; Anne et al. 2007). Despite this apparent lack of Sm protein sDMA modification, homozygous dart5 mutants are viable and, unlike hypomorphic mutations in Smn (Rajendra et al. 2007), do not display SMA-like phenotypes (Gonsalvez et al. 2006). Biochemically, the mutants expressed wild-type (WT) levels of the major Sm-class snRNPs (Gonsalvez et al. 2006). Thus, it appears that Dart5 is not critical for snRNP assembly in Drosophila. However, flies also express an ortholog of PRMT7, called Dart7 (CG9882; Boulanger et al. 2004). We therefore sought to determine whether methylation of Sm proteins was completely dispensable for snRNP assembly in Drosophila. Using RNA interference (RNAi), we found that Dart7 is required for Sm protein sDMA modification. Unlike dart5, dart7 is an essential gene. The lethal phenotype is unlikely to be caused by snRNP assembly defects, as the depleted animals contained residually methylated Sm proteins. Furthermore, the Dart7associated lethality could neither be rescued by overexpressing Dart5, nor could it be worsened by concomitant loss of Dart5. Thus, the two methyltransferases do not genetically interact. In contrast, dart5 and Smn mutants displayed a very strong genetic interaction. Notably, snRNP levels in the double mutants were unaffected. To conclusively test the requirement for methylation in snRNP assembly, we constructed fly strains expressing two mutant isoforms of $\mathrm{SmD1}$, one in which the entire C-terminal arginine-glycine $(\mathrm{RG})$-rich domain was deleted $(\Delta \mathrm{RG})$ and another in which the arginine residues were mutated to lysine (R-K). Neither isoform of SmD1 can be sDMA modified in vivo. Importantly, expression of SmD1(R-K) complemented a lethal SmD1 mutant allele. Furthermore, snRNP assays on the SmD1(R-K) adults indicated that Sm core assembly, TMG capping, and nuclear import were unaffected. Thus, symmetric dimethylation of C-terminal arginine residues in $\mathrm{SmD1}$ is required neither for viability nor snRNP assembly in Drosophila. In contrast to the R-K mutant, the $\Delta \mathrm{RG}$ transgene was unable to complement the lethality of the SmD1 mutant allele. Therefore, the C-terminal $\mathrm{RG}$ domain of SmD1 is required for organismal viability.

\section{RESULTS}

\section{Dart5 and Dart7 are required for $\mathrm{Sm}$ protein sDMA modification}

In human cells, Sm proteins are methylated in vivo by two different type II methyltransferases, PRMT5 and PRMT7 (Gonsalvez et al. 2007). We previously demonstrated that the Drosophila ortholog of PRMT5, Dart5, is required for Sm protein sDMA modification (Gonsalvez et al. 2006). In order to test for functional conservation between the two species, we assayed whether Dart7, the Drosophila ortholog of PRMT7, was also required for $\mathrm{Sm}$ protein methylation in vivo (Fig. 1). Schneider (S2) cells were depleted of either Dart5 or Dart7 using RNAi. Subsequently, a GFP-SmB reporter plasmid was transfected into the cells. The methylation status of GFP-SmB was analyzed using Y12, an antisDMA antibody (Brahms et al. 2000). Consistent with our findings in human cells, Sm protein methylation in Drosophila requires both Dart5 and Dart7 (Fig. 1B). We next sought to determine whether the two methyltransferases could functionally compensate for each other. S2 cells depleted of Dart5 were transfected with a myc-Dart7 overexpression plasmid, and cells depleted of Dart7 were transfected with myc-Dart5. The methylation status of GFP-SmB was analyzed in these cells (Fig. 1C). Neither methyltransferase could functionally compensate for loss of the other with respect to GFP-SmB methylation. We therefore conclude that Dart5 and Dart7 function nonredundantly in the Sm protein methylation pathway.

\section{dart7 is an essential gene}

We next sought to determine whether dart7 was required for viability. Since there are currently no publicly available gene-disruption stocks for dart7, we obtained a fly strain that expresses double-stranded (ds) RNA targeting the 
A

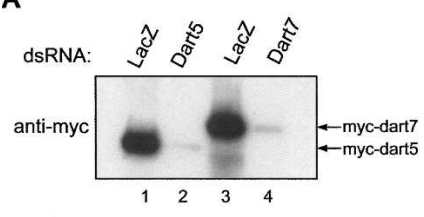

B

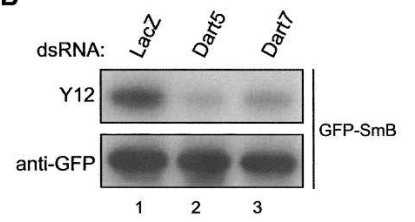

C

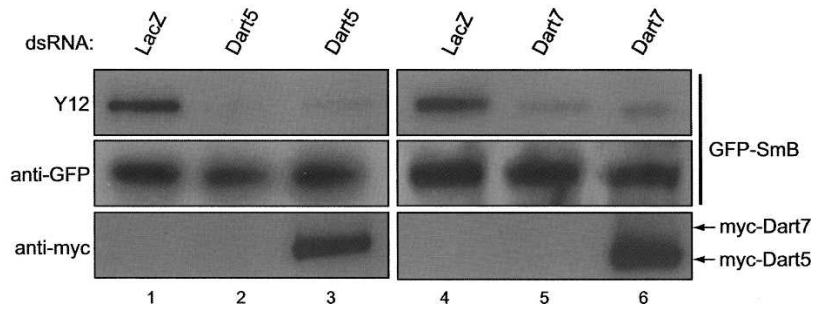

FIGURE 1. Dart5 and Dart7 are required for Sm protein sDMA modification. (A) Drosophila Schneider 2 (S2) cells were transfected with a plasmid expressing myc-Dart5 (lanes 1,2 ) or myc-Dart7 (lanes $3,4)$. The cells were subsequently incubated with dsRNAs targeting dart5 (lane 2), dart7 mRNA (lane 4), or lacZ (as a control, lanes 1,3). Lysates were prepared $72 \mathrm{~h}$ post-transfection, and the abundance of myc-Dart5 and myc-Dart7 was analyzed by Western blotting. The RNAi treatments efficiently depleted the target protein. (B) S2 cells were incubated with dsRNA targeting dart5 (lane 2) or dart7 (lane 3) mRNA. As a control, cells were also incubated with lacZ dsRNA (lane 1). Cells were again treated with the same dsRNA on day 3. On day 4 , a plasmid expressing GFP-SmB was transfected into the cells. On day 6 , the cells were harvested and lysates were prepared. The methylation status of GFP-SmB was examined by Western blotting using Y12 (top blot). The load was verified by stripping and reprobing the same blot with an anti-GFP antibody (bottom blot). In the presence of dart5 or dart7 dsRNA, the sDMA modification of GFP-SmB was reduced. $(C)$ S2 cells were incubated with dsRNA targeting dart5 (lanes 2,3) or dart7 (lanes 5,6) mRNA. As a control, cells were also incubated with lacZ dsRNA (lanes 1,4). The cells were re-treated with dsRNA as described in panel $B$, and GFP-SmB was transfected into the cells. In addition to GFP-SmB, the cells in lane 3 were transfected with a plasmid overexpressing myc-Dart7, and the cells in lane 6 were transfected with a plasmid overexpressing myc-Dart5. The cells in lanes 1,2, 4, and 5 were transfected with a control plasmid. Lysates were prepared on day 6 , and the methylation status of GFP-SmB was analyzed using Y12 (top blot). The blot was stripped and successively probed for GFP (middle blot) and myc (lower blot). Note that neither Dart5 nor Dart7 can functionally compensate for loss of the other with respect to GFP-SmB methylation.

dart7 mRNA (NIG stock center, Japan). Expression of dart7 dsRNA is under control of the GAL4-UAS system (Fig. 2A). Thus, depending on the choice of Gal4 driver, Dart7 can be depleted throughout the entire organism or depleted specifically from certain tissues (for review, see Duffy 2002). For this experiment, we depleted Dart7 in the entire organism by driving expression of Gal4 using the daughterless promoter (da-GAL4) (Bloomington Stock Center; donor J.-M. Dura, Institut de Génétique Humaine, Montpellier, France). Interestingly, this treatment resulted in pupal lethality. The Dart7 depleted animals survived to pupation but never emerged from their pupal cases (Fig. 2D). We next assayed the extent of dart7 depletion in these animals. Because antibodies that detect Dart7 are unavail- able, we were not able to determine the level of residual Dart7 protein in these animals. We therefore analyzed dart7 mRNA levels using semiquantitative RT-PCR. For this experiment, animals expressing dsRNA were analyzed shortly after the onset of pupation (i.e., a few hours prior to their death). We found that the level of dart7 mRNA
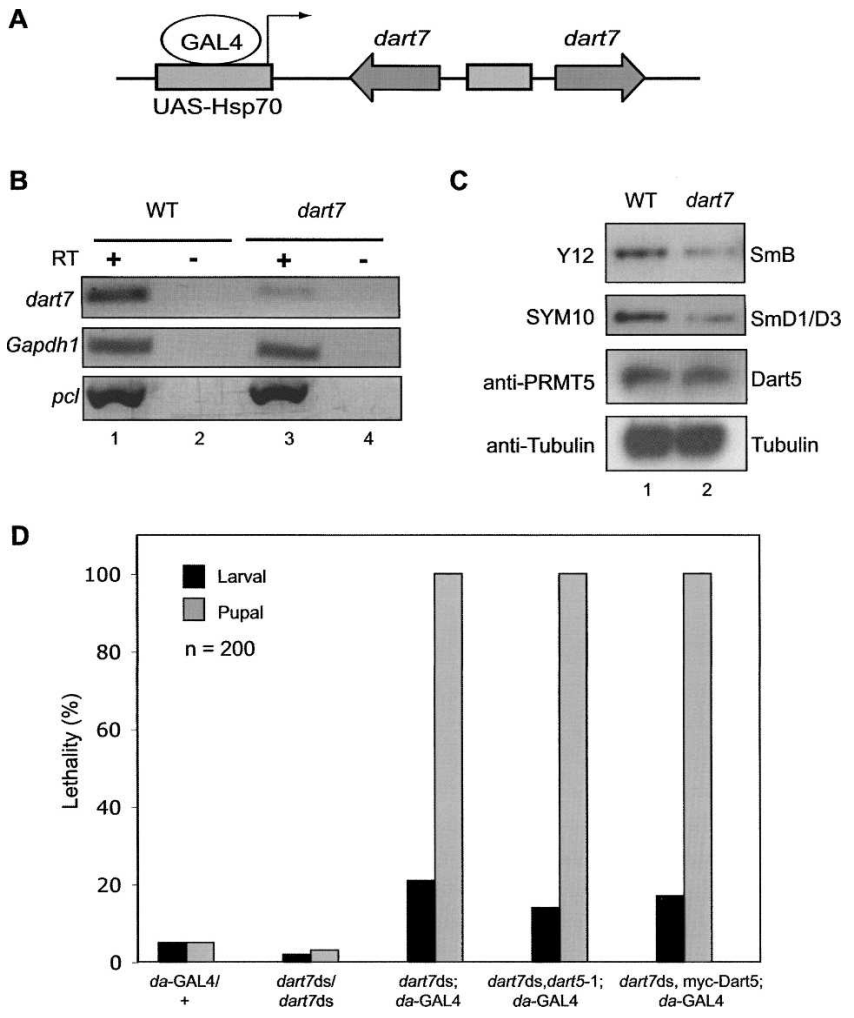

FIGURE 2. dart7 is an essential gene that does not genetically interact with dart5. (A) The genomic organization of the dart7 dsRNA strain is indicated. The expression of dart7 dsRNA is under the control of Gal4-responsive Upstream Activating Sequences (UAS). The dart7 sequences are present in an inverted repeat. Transcription from this locus results in the production of a hairpin duplex mRNA that is processed into siRNAs that are capable of mediating the RNAi response. (B) RNA was extracted from wild-type (WT; lanes 1,2) and Dart7-depleted pupae (lanes 3,4). The abundance of dart7 mRNA was analyzed using RT-PCR (top gel). The abundance of Gapdh1 (middle gel) and pcl (bottom gel) were monitored as loading controls. In order to ensure that the elongation products resulted from mRNA rather than contaminating DNA, reverse transcriptase was omitted from the reaction (lanes 2,4 ). The level of dart7 mRNA was drastically reduced in the dsRNA-expressing strain. $(C)$ Protein lysates were prepared from wild-type (WT; lane 1) and Dart7-depleted pupae (lane 2). The lysates were probed with the indicated antibodies (left). The detected proteins are also listed (right). Note that in the Dart7depleted lysates, Sm protein sDMA modification was reduced (Y12 and SYM10 blot). The level of Dart5, however, was unaffected (PRMT5 blot). (D) The viability of the indicated fly strains was analyzed. The flies were incubated for the course of the experiment at $26^{\circ} \mathrm{C}$. Note that depletion of Dart7 (dart7 ds; da-GAL4) was lethal primarily at the pupal phase. Depleting both Dart7 and Dart5 (dart7 ds, dart5-1; da-GAL4) from the same organism did not worsen the lethal phenotype. In addition, overexpression of myc-Dart5 in the Dart7 (dart7 ds, myc-Dart5; da-GAL4)-depleted animals was not able to restore viability. 
was considerably reduced in the dsRNA-expressing strain (Fig. 2B, top panel). Expression of a housekeeping gene (Gapdh1) was monitored and found to be unaffected (Fig. 2B, middle panel). Among the 482 different 21-nucleotide (nt) dsRNAs that could possibly be produced by the dart7 dsRNA transgene, only one of these 21 -mers was a perfect match to any other genomic target. The single perfectmatch 21-mer was within the pcl gene (CG5109). Thus, $p c l$ might be a possible off-target gene that could also be downregulated under these conditions. However, RT-PCR analysis indicated that, like Gapdh1, pcl mRNA levels are unaffected in the Dart7 depleted samples (Fig. 2B, bottom panel). Thus, the lethal phenotype can be attributed specifically to loss of Dart7. Lysates from Dart7-depleted pupae were further analyzed by Western blotting with Y12 and SYM10 antibodies. As with the RNAi experiments in S2 cells, we found that Sm protein sDMA modification was reduced in the Dart7-depleted flies (Fig. 2C). Dart5 levels, however, were unchanged (Fig. 2C). We conclude that the effect of Dart7 on Sm protein sDMA modification is direct.

The finding that dart7, but not dart5, is required for viability raises some interesting questions. For instance, can we restore viability in the Dart7-depleted animals by overexpressing Dart5? We tested this hypothesis by overexpressing a myc-Dart5 transgene in the Dart7 depleted background (Fig. 2D). The myc-Dart5 transgene used for this experiment is known to be functional, since it is capable of rescuing dart5 loss-of-function phenotypes (Gonsalvez et al. 2006). Consistent with the idea that the two enzymes function independently in substrate methylation, overexpression of myc-Dart5 failed to rescue the Dart7 associated lethality. In a complementary experiment, we found that disruption of Dart5 activity in Dart7depleted animals did not worsen the lethal phenotype (Fig. 2D). Thus, Dart5 and Dart7 are not functionally redundant.

\section{Dart5 is essential for viability when the level of $\mathrm{dSMN}$ is reduced}

Loss of Dart5 activity produces viable flies with no neuromuscular dysfunction. Although the mutants are defective in germline specification (Gonsalvez et al. 2006), it is unlikely that this phenotype is due to defects in snRNP biogenesis. In order to determine whether Dart 5 mediated $\mathrm{Sm}$ protein methylation is completely dispensable for snRNP assembly, we analyzed the viability of dart5 mutants under conditions where dSMN protein was limiting. For this experiment, a recently characterized $S m n$ hypomorphic allele $\left(S m n^{E 33}\right)$ was used (Rajendra et al. 2007). In $S m n^{E 33}$ mutants, dSMN levels are markedly reduced in the adult thorax. Whereas the dart5-1 and $S m n^{E 33}$ mutant alleles were homozygous viable when propagated independently, the double homozygotes all died when propagated on the same genetic background (Fig. 3A). Unexpectedly, signif-

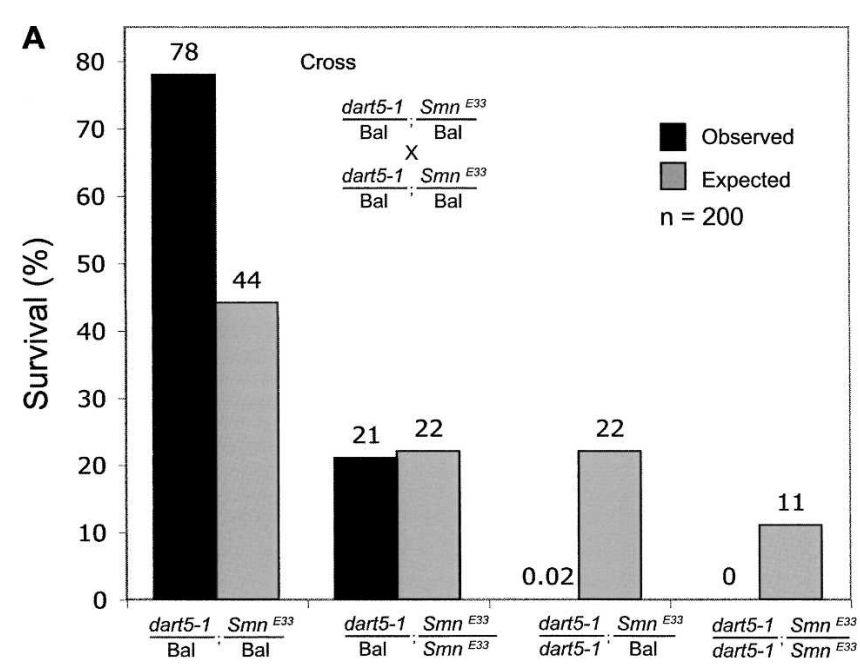

B

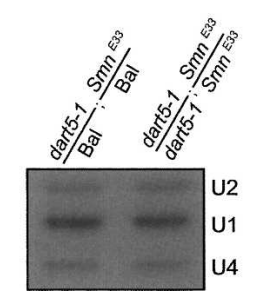

FIGURE 3. Smn and dart5 interact genetically. (A) The viability of the indicated fly strains was analyzed. The cross, as well as the resulting analysis, was performed at room temperature. Note that although the dart5-1, $S m n^{E 33}$ double heterozygotes are viable, no viable adult dart5-1, Smn ${ }^{E 33}$ double homozygotes were recovered. These two mutations therefore display a very strong genetic interaction. (B) RNA was extracted from dart5-1, Smn ${ }^{E 33}$ double homozygote pupae prior to their death (lane 2). As a control, RNA was also extracted from dart5-1, Smn ${ }^{E 33}$ double heterozygote pupae (lane 1). The RNA was examined using Northern blot analysis with the indicated probes. Despite the lethality of the dart5-1,Smn ${ }^{E 33}$ double homozygotes, the levels of the indicated snRNAs in these pupae were unaffected.

icant lethality was also observed for animals that were heterozygous for $S m n^{E 33}$ and homozygous for dart5-1. This was surprising because $S m n^{E 33}$ heterozygotes apparently produce wild-type amounts of dSMN (Rajendra et al. 2007). The lethal phase for both of these genotypes was during pupation.

To investigate whether the combined loss of Dart5 and dSMN resulted in defects in snRNP biogenesis, total RNA was extracted from the double mutants during the phenocritical stage and analyzed by Northern blotting. Despite the strong synthetic lethality associated with the dart5-1; $S m n^{E 33}$ double homozygotes, the overall levels of the major spliceosomal snRNAs were unaffected (Fig. 3B).

\section{Methylation of $\mathrm{Sm}$ proteins is not required for Sm-core assembly}

We have shown that Dart5 and Dart7 are each required for sDMA modification of Sm proteins in vivo (Fig. 1). 
However, both enzymes could potentially methylate many different substrates, thus making it difficult to correlate a particular organismal phenotype with defects observed on a molecular level. In order to conclusively test whether methylation of $\mathrm{Sm}$ proteins is required for snRNP assembly in flies, we made a series of GFP-SmD1 constructs. In addition to the wild-type control, two mutant transgenes were constructed, one in which the entire C-terminal RG tail was deleted $(\mathrm{SmD} 1 \Delta \mathrm{RG})$ and another in which the arginine residues within the tail were changed to lysine [SmD1(R-K)], see Figure 4A. Unlike arginine, lysine is not a substrate for Dart5 or Dart7 and, because it contains a primary amino group, it cannot be symmetrically dimethylated. To begin our molecular characterization, these three constructs were expressed in S2 cells and analyzed by fluorescence microscopy (Fig. 4B). As expected, WT GFPSmD1 localized primarily in the nucleus. Interestingly, SmD1(R-K) was also predominantly nuclear (Fig. 4B). In contrast, SmD1 $\Delta$ RG displayed a similar localization pattern to GFP alone, localizing to both the nucleus and the cytoplasm.

We next analyzed whether the mutant SmD1 proteins could interact with dSMN in a coimmunoprecipitation assay (Fig. 4C). Symmetric dimethylation of Sm proteins was previously shown to enhance binding to the Tudor domain of SMN (Brahms et al. 2001; Friesen et al. 2001). Although GFP-SmD1 associated efficiently with FlagdSMN, both mutant constructs (R-K and $\Delta \mathrm{RG}$ ) displayed reduced binding (Fig. 4C). Curiously, despite their distinct localization patterns [GFP-SmD1(R-K) was similar to wild type and GFP-SmD1DRG was pan-cellular], both constructs were similarly inefficient in binding to Flag-dSMN. Equivalent results were obtained by reversing the immunoprecipitation (data not shown).

To test the SmD1 constructs for functionality, we created transgenic fly strains that express Venus fluorescent protein (VFP) fusions under the control of the GAL4-UAS system. VFP is a derivative of yellow fluorescent protein (YFP) that is more stable for cell-biological applications (Nagai et al. 2002). Importantly, for biochemical applications, the VFP tag is recognized quite efficiently by anti-GFP antibodies. Three fly strains were created: VFP-SmD1, VFP-SmD1(R$\mathrm{K})$, and VFP-SmD1 $\Delta$ RG. Each of the tagged SmD1 constructs, along with a constitutive Tubulin driver (Tub-GAL4), were crossed onto the background of a lethal mutation in the endogenous smd1 gene (CG10753; snRNP69D). The allele contains a transposon insertion within the first intron of the snRNP69D locus (Fig. 5A). A detailed characterization of the mutants revealed that most of the homozygotes die during larval stages (Supplemental Fig. S1A). Surprisingly, a few homozygotes survive for up to $2 \mathrm{wk}$ as small larvae (note that WT flies typically pupate within $4-5 \mathrm{~d}$ ).

We carried out complementation analysis to determine if the three VFP-SmD1 transgenic lines could rescue the lethal phenotype of the mutant allele. Males bearing the

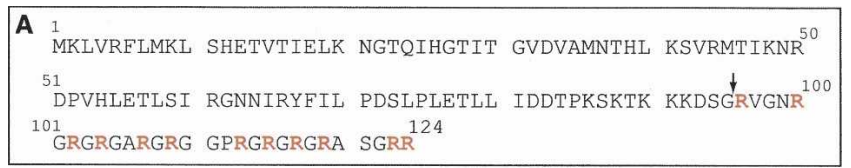

$\mathbf{B}$

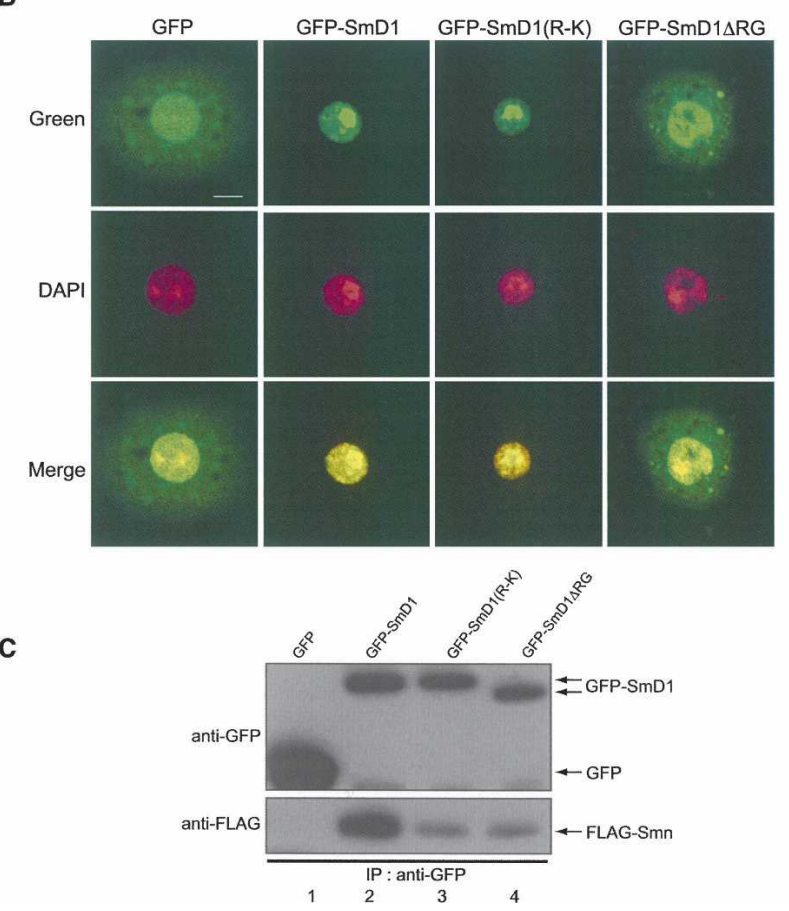

FIGURE 4. Methylation-negative mutants of SmD1 do not efficiently associate with SMN. (A) The protein sequence of SmD1 is indicated. (Arrow) Position of the truncation in the SmD1 RR construct. A stop codon was introduced at this position. The arginine residues indicated in red were site-specifically mutated to lysine to create the SmD1(R-K) mutant. (B) The following constructs were transfected into S2 cells: pAGW (GFP vector), pAGW-SmD1 (GFP-SmD1), pAGW-SmD1(R-K) (GFP-SmD1 R-K), and pAGW-SmD1 $\Delta$ RG (GFP-SmD1 $\Delta R G)$. The expression of the GFP constructs is under the control of the Act5c promoter. Note that while GFP and GFPSmD1 $\Delta R G$ display a pan-cellular distribution with slight nuclear enrichment, GFP-SmD1 and GFP-SmD1(R-K) localize almost exclusively within the nucleus. DAPI staining (pseudocolored magenta) indicates the position of the nucleus. $(C) \mathrm{S} 2$ cells were transfected with the following constructs: (lane 1) pAGW (GFP vector) and pAFWdSMN (Flag-dSMN), (lane 2) pAGW-SmD1 and pAFW-dSMN, (lane 3) pAGW-SmD1(R-K) and pAFW-dSMN, (lane 4) pAGW$\mathrm{SmD} 1 \Delta \mathrm{RG}$ and pAFW-dSMN. Expression of Flag-dSMN is under the control of the Act5c promoter. $72 \mathrm{~h}$ post-transfection, the cells were harvested and lysates were prepared. The GFP-tagged constructs were immunoprecipitated using polyclonal anti-GFP antibodies. The immunoprecipitates were probed using anti-Flag (bottom blot) and anti-GFP (top blot) antibodies. Note that both GFP-SmD1(R-K) and GFP-SmD1 $\Delta$ RG display reduced binding to SMN in comparison to wild type.

transposon insertion in $s n R N P 69 D$ and the appropriate VFP-transgene were crossed to females bearing the same insertion allele and the Tub-Gal4 driver. Since the snRNP69D allele is recessive lethal, both strains also contained a balancer chromosome. If the transgenes were able to completely rescue the mutant allele, $50 \%$ of the resulting 
A

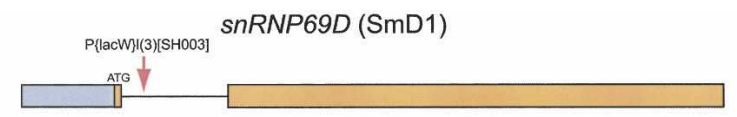

B
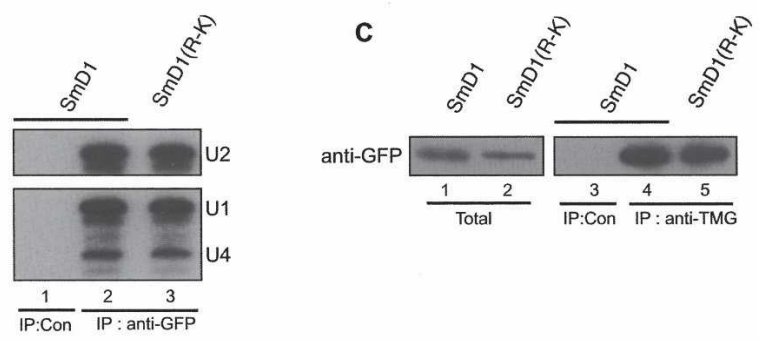

D
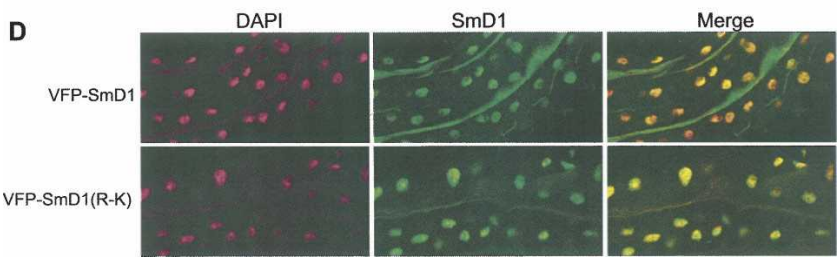

FIGURE 5. SnRNP assembly and viability are unaffected in $\mathrm{SmD} 1$ (R$\mathrm{K})$ mutants. (A) The smd1 locus (CG10753; also called snRNP69D) is depicted. The position of the $\mathrm{P}$ element ( $\mathrm{P}\{\mathrm{lacW}\} 1(3)$ [SH003], obtained from the Szeged Drosophila Stock Center) used in this study is indicated (arrow). (B) Lysates were prepared from adult flies expressing either VFP-SmD1 (lanes 1,2) or VFP-SmD1(R-K) (lane $3)$. Since the transgenes are expressed on a homozygous mutant snRNP69D ${ }^{\mathrm{P}\{\mathrm{lacW}\} 1(3)[\mathrm{SH} 003]}$ background, endogenous $\mathrm{SmD1}$ is not expressed in these strains. The lysates were subjected to immunoprecipitation with polyclonal anti-GFP antibodies (lanes 2,3), or as a control for binding, protein A beads with no bound antibody (lane 1). The bound RNAs were extracted from the immunoprecipitates, purified, and analyzed by Northern blotting using the indicated snRNA probes. Wild-type and mutant SmD1 constructs associate with equivalent amounts of snRNA in vivo. Thus, Sm core assembly is not affected in the SmD1(R-K) strain. $(C)$ Lysates were prepared from adult flies expressing either VFP-SmD1 (lanes 1,3,4) or VFP-SmD1(R$\mathrm{K})$ (lanes 2,5). The lysates were subjected to immunoprecipitation with anti-TMG antibody-coated beads (lanes 4,5), or as a control, protein A beads with no bound antibody (lane 3). The immunoprecipitates (lanes 3-5) as well as representative fractions from the total lysates (lanes 1,2 ) were analyzed by Western blotting using a monoclonal anti-GFP antibody. The snRNAs present in the wild-type and mutant SmD1 strains are TMG capped. (D) Gut tissue was dissected and fixed from flies expressing either VFP-SmD1 (top panel) or VFPSmD1(R-K) (bottom panel). Both wild-type and mutant SmD1 (green) localize to the nucleus. DAPI staining (magenta) indicates the position of the nuclei in these cells. Thus, as with $\mathrm{Sm}$ core assembly and TMG capping, nuclear import of snRNPs is also unaffected in the SmD1(R-K) background.

progeny from this cross should have been homozygous for the mutant snRNP69D allele (the remainder would be heterozygous and contain the balancer chromosome). Such a result would indicate that the VFP-transgenes can functionally compensate for loss of endogenous SmD1. We found that both VFP-SmD1 and -SmD1(R-K) were able to partially complement the lethal phenotype, producing viable adults. However, only $\sim 30 \%$ of the progeny were homozygous for the allele (Supplemental Fig. S1B). Thus, the rescue was incomplete (i.e., even for the WT transgene), perhaps due to the presence of the VFP tag. Alternatively, the partial complementation might be due to problems with the expression profile of the Tub-Gal4 driver. In the latter scenario, the driver might not be expressed as strongly as the endogenous snRNP69D promoter or might not be expressed at appropriate levels in all relevant tissues. In the case of both transgenes, however, almost all of the homozygous flies that pupated were able to reach adulthood (Supplemental Fig. S1B). In contrast to VFP-SmD1 and -SmD1(R-K), the VFP-SmD1 1 RG strain did not produce viable adults (Supplemental Fig. S1A). It should be noted, however, that the SmD1 $\Delta \mathrm{RG}$ transgene retains partial functionality, as the lethal phase of homozygous snRNP69D mutants was significantly delayed. Moreover, the larvae expressing SmD1 $\Delta \mathrm{RG}$ were considerably larger than the parental snRNP69D line (Supplemental Fig. S1A).

To examine whether snRNP assembly was affected in the SmD1(R-K) mutants, we performed an Sm core assay (Fig. $5 \mathrm{~B})$. Lysates were prepared from adult flies expressing either VFP-SmD1 or -SmD1(R-K) and subjected to immunoprecipitation using polyclonal anti-GFP antibodies. The precipitated RNAs were extracted and analyzed by Northern blotting. Equivalent levels of snRNAs were associated with each construct. Thus, Sm core assembly is not affected in the sDMA-negative SmD1(R-K) strain. We also analyzed trimethylguanosine (TMG) capping in these strains (Fig. 5C). The TMG cap modification is found exclusively on snRNPs that have undergone proper Sm core assembly and is a prerequisite for nuclear import (Hamm et al. 1990). Lysates were prepared, and coimmunoprecipitation analysis was performed using anti-TMG-coupled agarose beads. The immunoprecipitates were analyzed by Western blotting to determine the level of incorporation of VFP-tagged SmD1 into mature snRNPs. Equivalent levels of VFPSmD1 and VFP-SmD1(R-K) were precipitated using this assay (Fig. 5C), suggesting that the mutants contain properly assembled, TMG-capped snRNPs. Finally, we prepared tissue from adult flies and analyzed the subcellular localization of VFP-SmD1 and -SmD1(R-K). Consistent with our results in S2 cells, both the wild-type construct and the VFP-SmD1(R-K) protein were predominantly nuclear (Fig. 5D). Because SmD1(R-K) is incapable of being sDMA modified and its expression complements the loss of endogenous $\mathrm{SmD1}$, we conclude that symmetric arginine dimethylation of Sm proteins is not required for snRNP assembly in Drosophila.

\section{DISCUSSION}

SMN is a central player in the snRNP biogenesis pathway and is thought to contribute to the specificity of RNP assembly by ensuring the loading of Sm proteins onto the appropriate RNAs (Pellizzoni et al. 2002). In human cells, the sDMA modification present on Sm proteins mediates 
efficient association with the SMN complex. Consequently, loss of this post-translational modification results in defects in snRNP assembly (Gonsalvez et al. 2007). Here, we have analyzed whether methylation of Sm proteins is a critical determinant of snRNP assembly in Drosophila.

We demonstrated that, similar to the situation in human cells, Drosophila Sm proteins are methylated by two enzymes-Dart5 and Dart7 (Fig. 1). Both enzymes are required for full Sm protein sDMA modification in vivo, and neither enzyme can functionally compensate for loss of the other. The precise mechanism by which Dart5 and Dart7 function to methylate Sm proteins is not known. We envision at least two distinct mechanisms by which these proteins could function. One model suggests that each enzyme methylates distinct residues on Sm proteins, such that full Y12 or SYM10 reactivity requires the enzymatic activity of both proteins. Partial methylation of the Sm protein by either Dart5 or Dart7 would not be sufficient for Y12 or SYM10 recognition. In support of this model, SYM10 antibodies require at least two sDMA residues for efficient recognition (Boisvert et al. 2002); the exact Y12 epitope is not known. An alternate model suggests that one enzyme modulates the activity of the other. In this scenario, only Dart5 or Dart7 would participate directly in Sm protein methylation, while the other enzyme would function as a regulator. Experiments aimed at distinguishing between these models are ongoing.

Although dart5 is not required for organismal viability, dart7 is an essential gene (Fig. 2). Flies depleted of Dart7 protein die during pupation. Our data further suggest that the lethal phenotype is not likely to be due to defects in snRNP assembly, as the anti-sDMA reactivity of Sm proteins was reduced, but not eliminated, in the depleted lysates (Fig. 2C). Furthermore, in the viable dart5 mutants, no Y12- or SYM10-reactive Sm proteins were detected (Gonsalvez et al. 2006). Additionally, null mutants with defects in snRNP assembly factors typically die during embryonic or larval stages (e.g., SmD1, SmD3, SmB, SmE, TGS1, and SMN). Finally, flies that exclusively express an SmD1 isoform that cannot be symmetrically dimethylated are viable. Based on these observations, we conclude that the Dart7-depleted flies most likely die due to aberrant methylation of a non-Sm target.

In contrast to dart7, dart5 null and Smn hypomorphic mutants display a very strong synthetic lethal interaction. Significant lethality was observed even under conditions where the dart5 mutant allele was homozygous and the Smn allele was heterozygous. This was a surprising finding since we previously reported that the Smn heterozygotes produced wild-type amounts of dSMN (Rajendra et al. 2007). However, in this same mutant line $\left(S m n^{E 33}\right)$, a decrease in dSMN levels was apparent only in the adult thorax of homozygous animals. Since the $S m n^{E 33}$ allele contains a mutation in the Smn promoter, it is possible that dSMN levels are reduced in other uncharacterized tissues in homozygous and heterozygous $S m n^{E 33}$ flies. This would explain the very strong synthetic lethality that is observed in combination with a loss-of-function dart5 mutant. Despite the strong genetic interaction, however, the snRNP levels in the double mutants were unaffected. Based on this finding, we conclude that the double mutants either die from loss of a non-snRNP related function, or that snRNP assembly is reduced in certain critical tissues (for example, neurons), which in turn results in organismal lethality. If the latter explanation is true, depending on the relative mass of the sensitive tissue in comparison to the entire organism, total snRNA levels might appear to be unaffected. In considering this result, it is important to note that the overall levels of snRNAs were also unaffected in Smn null mutants, which die during larval stages (Rajendra et al. 2007).

To conclusively test the requirement for Sm protein methylation in snRNP assembly, we constructed fly strains expressing two mutant isoforms of SmD1. We chose SmD1 as the focus of this study for three reasons. First, unlike $\mathrm{SmB}$ and SmD3, SmD1 does not form part of the U7 snRNP (Pillai et al. 2001). As such, the phenotypes we observed can be attributed specifically to spliceosomal snRNPs. Second, prior to Sm core assembly, SmD1 exists in a subcomplex with SmD2 (Raker et al. 1996). Thus, mutation of SmD1 could potentially inhibit incorporation of the entire D1/D2 subcomplex into the Sm core. In contrast, $\mathrm{SmB}$ and $\mathrm{SmD} 3$ form a separate subcomplex (Raker et al. 1996). Thus, even if we were to disrupt the methylation of one of these proteins, the other would still be methylated and could diminish the severity of the phenotype. Third, truncation of the C-terminal tail of Saccharomyces cerevisiae $\mathrm{SmD} 1$ produced a more severe phenotype than truncation of the $\mathrm{C}$ termini of either $\mathrm{SmB}$ or SmD3 (Zhang et al. 2001). This suggests that the RG tail of SmD1 plays a critical role in metazoan snRNP assembly. Consistent with this earlier finding in $S$. cerevisiae, the SmD1 $\Delta R G$ isoform was unable to rescue the lethal phenotype of an SmD1 mutant allele. Interestingly, however, the sDMA-negative $\mathrm{SmD} 1(\mathrm{R}-\mathrm{K})$ isoform was able to complement the lethal phenotype of the SmD1 mutant. Assays on the SmD1(R-K) flies demonstrated that Sm core assembly, TMG capping, and snRNP import into the nucleus were each unaffected in comparison to the WT SmD1 transgene. Thus, sDMA modification of SmD1 is not required for snRNP assembly in Drosophila.

A caveat of these studies is that $\mathrm{SmB}$ and $\mathrm{SmD} 3$ remain methylated in the mutant $\mathrm{SmD} 1(\mathrm{R}-\mathrm{K})$ strain. Therefore, it is a formal possibility that methylated $\mathrm{SmB}$ and SmD3 are able to overcome the need for SmD1 methylation in snRNP assembly. However, if the SmD1(R-K) results are considered in the context of the dart 5 mutant phenotype, the most logical conclusion is that Sm protein sDMA modification in Drosophila is not essential for snRNP assembly. In order to conclusively prove this 
point, however, three mutant alleles (loss-of-function mutants of $\mathrm{SmB}, \mathrm{SmD1}$, and $\mathrm{SmD3}$ ) and three transgenes (methylation negative mutants of SmB, SmD1, and SmD3) would all need to be expressed in the same genetic background.

Given that the SmD1(R-K) construct was viable with no apparent defects in snRNP assembly, it was somewhat surprising that the mutant displayed reduced binding to dSMN (Fig. 4C). This suggests that, similar to the situation in human cells, Drosophila SMN preferentially associates with sDMA-modified Sm proteins. This result is consistent with our previous finding that Dart5 depletion results in reduced SMN-SmB association (Gonsalvez et al. 2006). Importantly, dart5 null mutants are viable and express normal levels of snRNPs. It is clear that dSMN is required for efficient snRNP assembly in Drosophila (Rajendra et al. 2007). However, it is also possible that, upon disruption of efficient SMN-Sm binding, backup mechanisms ensure proper and continued snRNP assembly.

In conclusion, our results suggest that the mechanism of snRNP assembly in flies differs in certain respects from that of mammals. Although Sm proteins are sDMA modified in Drosophila by two methyltransferases, Dart5 and Dart7, these modifications do not appear to play a significant role in snRNP assembly. In contrast, Sm protein methylation is a critical requirement for mammalian snRNP biogenesis (Gonsalvez et al. 2007). With respect to snRNP assembly, other differences also exist between Drosophila and humans. Phosphorylation of N-terminal serine residues of human SMN is required for efficient activity (Grimmler et al. 2005). The $\mathrm{N}$ terminus of the human protein that contains the phospho-serine residues is not conserved in invertebrates (data not shown). Furthermore, the SMN protein exists in humans as part of a large oligomeric complex containing at least nine different proteins (Gemins2-8 and unrip). Bioinformatic analyses have yet to identify unambiguous orthologs of Gemins3-8 in Drosophila. We envision that, during evolution, layers of regulation have been added to the snRNP assembly pathway. In support of this theory, SMN itself is present in fission yeast genomes but is completely absent from budding yeast (Liu et al. 1997; Hannus et al. 2000). In fact, S. cerevisiae Sm proteins are not sDMA-modified, as they lack appropriate RG residues in their C-terminal tails (Rymond 1993; Roy et al. 1995). Thus, Drosophila represents an intermediate between budding yeast and mammals. In the fruit fly, the $\mathrm{Sm}$ proteins are methylated and SMN is required for snRNP assembly. However, the known post-translational modifications of SMN and Sm proteins are dispensable for snRNP assembly. The fact that Drosophila Sm proteins retain this methyl modification suggests an ancestral function that is unrelated to snRNP assembly. The available data indicate that methylation of Sm proteins is likely to be involved in specification of the germline (Gonsalvez et al. 2006; Anne et al. 2007).

\section{MATERIALS AND METHODS}

\section{DNA constructs}

GFP-SmB (Drosophila) was constructed using PCR and Gateway recombination (Invitrogen). A PCR product containing the $S m B$ open reading frame and the gateway recombination sequence were first cloned into the pDONR-221 entry vector (Invitrogen). Subsequently, SmB was recombined into the pAGW expression vector (Drosophila Genome Research Center, DGRC). This vector enables the expression of GFP-tagged proteins in S2 cells using the Actin $5 c$ promoter. A similar cloning strategy was used to clone WT SmD1, SmD1(R-K), and SmD1 $\mathrm{RG}$ into pAGW. The three SmD1 constructs were also cloned into the trangenesis vector, pPVW (DGRC). This vector enables expression of a Venus Fluorescent Protein (VFP)-tagged construct under the control of the GAL4 responsive Upstream Activation Sequence (UAS). Similar cloning strategies were also used to clone the dart5 and dart7 cDNAs into the pAMW vector (Actin5c driven myc-tagging vector, DGRC). The cDNA for $S m n$ was likewise cloned into pAFW (Actin5c driven Flag-tagging vector, DGRC).

\section{Cell culture and transfections}

Drosophila Schneider 2 (S2) cells were grown in SFM media (Invitrogen). DNA was transfected into S2 cells using the Cellfectin reagent (Invitrogen) as described by the manufacturer. For standard transfection experiments, the cells were analyzed $72 \mathrm{~h}$ post-transfection. Dart5 and Dart7 were depleted from S2 cells by incubating the cells in media containing dsRNA specific for the respective gene. For the GFP-SmB methylation experiment, cells were first treated with dsRNA to deplete Dart5 and Dart7. The cells were again treated with dsRNA on day 3 . On day 4 , the cells were transfected with the GFP-SmB plasmid. The cells were harvested and analyzed on day 6 .

\section{Lysate preparation and immunoprecipitations}

S2 cell lysates were prepared by resuspending the cells in RIPA buffer (50 mM Tris.Cl pH 7.5, $150 \mathrm{mM} \mathrm{NaCl,} \mathrm{1 \%} \mathrm{NP-40,} 1 \mathrm{mM}$ EDTA) containing protease inhibitors (Halt protease inhibitor cocktail kit, Pierce) and passing several times through a 25 guage needle. The lysate was cleared by centrifugation at $10,000 \mathrm{~g}$ for $10 \mathrm{~min}$ at $4^{\circ} \mathrm{C}$. Binding experiments, including subsequent wash steps, were conducted using gentle binding buffer $(50 \mathrm{mM}$ Tris $\mathrm{pH}$ 7.5, $200 \mathrm{mM} \mathrm{NaCl}, 0.2 \mathrm{mM}$ EDTA, and 0.05\% NP-40). The binding buffer contained protease inhibitors, and incubations were performed at $4^{\circ} \mathrm{C}$. Fly lysates were likewise prepared by homogenizing pupae using a small pestle in RIPA buffer containing protease inhibitors and clearing by centrifugation at $10,000 \mathrm{~g}$ for $10 \mathrm{~min}$ at $4^{\circ} \mathrm{C}$.

\section{Antibodies}

The RNAi knock-down efficiencies of myc-Dart5 and myc-Dart7 were analyzed using a polyclonal anti-myc antibody (SantaCruz Biotechnology, Inc.). For immunoprecipitation of Drosophila GFP-SmB, polyclonal antibodies (Abcam) were used.

The immunoprecipitated protein was subsequently analyzed using Y12 (kind gift of J. Steitz), and the load was verified using the monoclonal anti-GFP antibody (Roche). The same antibodies 
were used in the complementation experiment. The methylation status of Sm proteins in the Dart7 depleted lysates was analyzed using SYM10 (Upstate) and Y12. The anti-PRMT5 antibody was obtained from Upstate, and the anti-Tubulin antibody was obtained from Sigma Aldrich. In the SmD1/dSMN interaction experiment, GFP-tagged SmD1 was immunoprecipitated using polyclonal GFP antibodies. The precipitates were subsequently probed using a monoclonal Flag antibody (Sigma Aldrich) and a monoclonal GFP antibody. In the Sm-core assembly assay, polyclonal GFP antibodies were used for the immunoprecipitation. For the TMG capping assay, anti-TMG antibody coated beads (EMD Biosciences, Inc.) were used in the immunoprecipitation. The precipitates were probed with the monoclonal GFP antibody.

\section{Imaging}

Immunofluorescence images were captured using a TCS SP2 laser scanning confocal microscope and assembled using Photoshop and Illustrator (Adobe). The Leica Confocal Scanner was interfaced with Leica Confocal Software.

\section{RNA extraction, RT-PCR, and Northern blotting}

RNA was extracted from wild-type and Dart7 depleted pupae using Trizol as directed by the manufacturer. The Superscript First Strand Synthesis kit (Invitrogen) was used for the RT-PCR reaction. The relative abundance of dart7 was analyzed using 25 cycles. The relative abundance of Gapdh1 and $p c l$ mRNAs was analyzed using 20 cycles. The cycle number was optimized to ensure that the PCR reaction was in the linear range. Northern blot probes were prepared using the Rediprime labeling kit (Amersham). Total RNA was run on a $10 \%$ polyacrylamide-urea gel (Invitrogen), transferred to a nylon membrane, and probed with ${ }^{32} \mathrm{P}$-labeled PCR products corresponding to the D. melanogaster U1, U2, and U4 snRNAs.

\section{Fly stocks}

Oregon- $\mathrm{R}$ was used as the wild-type strain. Unless otherwise specified, all balancer chromosomes and dominant markers used in this study were described previously (Lindsley and Zimm 1992). All stocks were cultured on standard cream of wheat-agar medium at room temperature $\left(25^{\circ} \mathrm{C} \pm 1^{\circ} \mathrm{C}\right)$ in half-pint bottles. Flies expressing GAL4 from the Tubulin promoter (Tub-GAL4) were a kind gift of M. Ramaswami (University of Arizona, Tucson, Arizona). The fly strain expressing dsRNA targeting the dart7 gene was obtained from the NIG-fly stock center (Japan). The da-GAL4 strain was obtained from the Bloomington Stock Center, and the snRNP69D mutant allele was obtained from the Szeged Drosophila Stock Center. The transgenic fly strains (myc-Dart5, VFP-SmD1, VFP-SmD1 [R-K], and VFP-SmD1 $\Delta \mathrm{RG}$ ) were injected and balanced by BestGene, Inc.

\section{Fly crosses}

The dsRNA fly crosses were performed by placing males and females of the appropriate genotype in embryo collection chambers. Virgin females were placed for $3 \mathrm{~d}$ at $26^{\circ} \mathrm{C}$ prior to mating to enhance production of GAL4. The mating, as well as all subsequent steps, were performed at $26^{\circ} \mathrm{C}$. The adults were removed after $16 \mathrm{~h}$ of mating. First instar larvae were sorted by genotype at $28 \mathrm{~h}$. The larvae were counted and transferred to new bottles containing fresh food. Fresh food was added to the bottle during the subsequent $3 \mathrm{~d}$, i.e., during the larval growth period. The viability of larvae and pupae were analyzed. The dart5-1; SmnE $E^{33}$ genetic interaction cross as well as the $\mathrm{SmD1}$ viability crosses were performed at room temperature.

\section{SUPPLEMENTAL DATA}

Supplemental material can be found at http://www.rnajournal.org.

\section{ACKNOWLEDGMENTS}

We thank J. Steitz and M. Ramaswami for providing reagents. We thank R. Ueda and the NIG stock center (Japan) for providing the dart7 dsRNA strain. We are especially grateful to T.K. Rajendra for insightful discussions throughout the course of this work and to all the members of the Matera laboratory for critical reading of the manuscript. This work was supported by N.I.H. grants R01GM053034 and R01-NS041617 (to A.G.M.). Microscopy support was provided by N.I.H. grants S10-RR021228 and S10-RR017980. G.B.G. was supported in part by NIH postdoctoral traineeships, T32-HD007104 and F32-HD055711.

Received November 27, 2007; accepted January 30, 2008.

\section{REFERENCES}

Anne, J., Ollo, R., Ephrussi, A., and Mechler, B.M. 2007. Arginine methyltransferase Capsuléen is essential for methylation of spliceosomal Sm proteins and germ cell formation in Drosophila. Development 134: 137-146.

Avila, A.M., Burnett, B.G., Taye, A.A., Gabanella, F., Knight, M.A., Hartenstein, P., Cizman, Z., Di Prospero, N.A., Pellizzoni, L., Fischbeck, K.H., et al. 2007. Trichostatin A increases SMN expression and survival in a mouse model of spinal muscular atrophy. J. Clin. Invest. 117: 659-671.

Bedford, M.T. and Richard, S. 2005. Arginine methylation an emerging regulator of protein function. Mol. Cell 18: 263-272.

Boisvert, F.M., Cote, J., Boulanger, M.C., Cleroux, P., Bachand, F., Autexier, C., and Richard, S. 2002. Symmetrical dimethylarginine methylation is required for the localization of SMN in Cajal bodies and pre-mRNA splicing. J. Cell Biol. 159: 957-969.

Boulanger, M.C., Miranda, T.B., Clarke, S., Di Fruscio, M., Suter, B., Lasko, P., and Richard, S. 2004. Characterization of the Drosophila protein arginine methyltransferases DART1 and DART4. Biochem. J. 379: 283-289.

Brahms, H., Raymackers, J., Union, A., de Keyser, F., Meheus, L., and Lührmann, R. 2000. The C-terminal RG dipeptide repeats of the spliceosomal Sm proteins D1 and D3 contain symmetrical dimethylarginines, which form a major B-cell epitope for anti-Sm autoantibodies. J. Biol. Chem. 275: 17122-17129.

Brahms, H., Meheus, L., de Brabandere, V., Fischer, U., and Lührmann, R. 2001. Symmetrical dimethylation of arginine residues in spliceosomal $\mathrm{Sm}$ protein $\mathrm{B} / \mathrm{B}^{\prime}$ and the Sm-like protein LSm4, and their interaction with the SMN protein. RNA 7: 1531-1542.

Carrel, T.L., McWhorter, M.L., Workman, E., Zhang, H., Wolstencroft, E.C., Lorson, C., Bassell, G.J., Burghes, A.H., and Beattie, C.E. 2006. Survival motor neuron function in motor axons is independent of functions required for small nuclear ribonucleoprotein biogenesis. J. Neurosci. 26: 11014-11022.

Duffy, J.B. 2002. GAL4 system in Drosophila: A fly geneticist's Swiss army knife. Genesis 34: 1-15. 
Friesen, W.J., Massenet, S., Paushkin, S., Wyce, A., and Dreyfuss, G. 2001. SMN, the product of the spinal muscular atrophy gene, binds preferentially to dimethylarginine-containing protein targets. Mol. Cell 7: 1111-1117.

Gabanella, F., Butchbach, M.E., Saieva, L., Carissimi, C., Burghes, A.H., and Pellizzoni, L. 2007. Ribonucleoprotein assembly defects correlate with spinal muscular atrophy severity and preferentially affect a subset of spliceosomal snRNPs. PLoS ONE 2: e921. doi: 10.1371/journal.pone.0000921.

Gonsalvez, G.B., Rajendra, T.K., Tian, L., and Matera, A.G. 2006. The Sm-protein methyltransferase, dart5, is essential for germ-cell specification and maintenance. Curr. Biol. 16: 1077-1089.

Gonsalvez, G.B., Tian, L., Ospina, J.K., Boisvert, F.M., Lamond, A.I., and Matera, A.G. 2007. Two distinct arginine methyltransferases are required for biogenesis of Sm-class ribonucleoproteins. J. Cell Biol. 178: 733-740.

Grimmler, M., Bauer, L., Nousiainen, M., Korner, R., Meister, G., and Fischer, U. 2005. Phosphorylation regulates the activity of the SMN complex during assembly of spliceosomal U snRNPs. EMBO Rep. 6: 70-76.

Hamm, J., Darzynkiewicz, E., Tahara, S.M., and Mattaj, I.W. 1990. The trimethylguanosine cap structure of U1 snRNA is a component of a bipartite nuclear targeting signal. Cell 62: 569-577.

Hannus, S., Buhler, D., Romano, M., Seraphin, B., and Fischer, U. 2000. The Schizosaccharomyces pombe protein Yab8p and a novel factor, Yiplp, share structural and functional similarity with the spinal muscular atrophy-associated proteins SMN and SIP1. Hum. Mol. Genet. 9: 663-674.

Huber, J., Cronshagen, U., Kadokura, M., Marshallsay, C., Wada, T., Sekine, M., and Lührmann, R. 1998. Snurportin1, an m3G-capspecific nuclear import receptor with a novel domain structure. EMBO J. 17: 4114-4126.

Jady, B.E., Darzacq, X., Tucker, K.E., Matera, A.G., Bertrand, E., and Kiss, T. 2003. Modification of Sm small nuclear RNAs occurs in the nucleoplasmic Cajal body following import from the cytoplasm. EMBO J. 22: 1878-1888.

Lefebvre, S., Burglen, L., Reboullet, S., Clermont, O., Burlet, P., Viollet, L., Benichou, B., Cruaud, C., Millasseau, P., Zeviani, M., et al. 1995. Identification and characterization of a spinal muscular atrophy-determining gene. Cell 80: 155-165.

Lindsley DaZ, G.G. and Zimm, G.G. 1992. The genome of Drosophila melanogaster. Academic Press, San Diego.

Liu, Q., Fischer, U., Wang, F., and Dreyfuss, G. 1997. The spinal muscular atrophy disease gene product, SMN, and its associated protein SIP1 are in a complex with spliceosomal snRNP proteins. Cell 90: 1013-1021.

Massenet, S., Pellizzoni, L., Paushkin, S., Mattaj, I.W., and Dreyfuss, G. 2002. The SMN complex is associated with snRNPs throughout their cytoplasmic assembly pathway. Mol. Cell. Biol. 22: 6533-6541.

Matera, A.G., Terns, R.M., and Terns, M.P. 2007. Noncoding RNAs: Lessons from the small nuclear and small nucleolar RNAs. Nat. Rev. Mol. Cell Biol. 8: 209-220.

Meister, G., Eggert, C., and Fischer, U. 2002. SMN-mediated assembly of RNPs: A complex story. Trends Cell Biol. 12: 472-478.

Mouaikel, J., Verheggen, C., Bertrand, E., Tazi, J., and Bordonne, R. 2002. Hypermethylation of the cap structure of both yeast snRNAs and snoRNAs requires a conserved methyltransferase that is localized to the nucleolus. Mol. Cell 9: 891-901.
Mouaikel, J., Narayanan, U., Verheggen, C., Matera, A.G., Bertrand, E., Tazi, J., and Bordonne, R. 2003. Interaction between the small-nuclear-RNA cap hypermethylase and the spinal muscular atrophy protein, survival of motor neuron. EMBO Rep. 4: 616-622.

Nagai, T., Ibata, K., Park, E.S., Kubota, M., Mikoshiba, K., and Miyawaki, A. 2002. A variant of yellow fluorescent protein with fast and efficient maturation for cell-biological applications. Nat. Biotechnol. 20: 87-90.

Narayanan, U., Ospina, J.K., Frey, M.R., Hebert, M.D., and Matera, A.G. 2002. SMN, the spinal muscular atrophy protein, forms a pre-import snRNP complex with snurportinl and importin-ß. Hum. Mol. Genet. 11: 1785-1795.

Narayanan, U., Achsel, T., Lührmann, R., and Matera, A.G. 2004 Coupled in vitro import of $U$ snRNPs and SMN, the spinal muscular atrophy protein. Mol. Cell 16: 223-234.

Nesic, D., Tanackovic, G., and Kramer, A. 2004. A role for Cajal bodies in the final steps of U2 snRNP biogenesis. J. Cell Sci. 117: 4423-4433.

Ohno, M., Segref, A., Bachi, A., Wilm, M., and Mattaj, I.W. 2000. PHAX, a mediator of U snRNA nuclear export whose activity is regulated by phosphorylation. Cell 101: 187-198.

Ospina, J.K., Gonsalvez, G.B., Bednenko, J., Darzynkiewicz, E., Gerace, L., and Matera, A.G. 2005. Cross-talk between snurportin1 subdomains. Mol. Biol. Cell 16: 4660-4671.

Paushkin, S., Gubitz, A.K., Massenet, S., and Dreyfuss, G. 2002. The SMN complex, an assemblyosome of ribonucleoproteins. Curr. Opin. Cell Biol. 14: 305-312.

Pellizzoni, L., Yong, J., and Dreyfuss, G. 2002. Essential role for the SMN complex in the specificity of snRNP assembly. Science 298: 1775-1779.

Pillai, R.S., Will, C.L., Lührmann, R., Schumperli, D., and Muller, B. 2001. Purified U7 snRNPs lack the Sm proteins D1 and D2 but contain Lsm10, a new $14 \mathrm{kDa}$ Sm D1-like protein. EMBO J. 20: 5470-5479.

Rajendra, T.K., Gonsalvez, G.B., Walker, M.P., Shpargel, K.B., Salz, H.K., and Matera, A.G. 2007. A Drosophila melanogaster model of spinal muscular atrophy reveals a function for SMN in striated muscle. J. Cell Biol. 176: 831-841.

Raker, V.A., Plessel, G., and Lührmann, R. 1996. The snRNP core assembly pathway: Identification of stable core protein heteromeric complexes and an snRNP subcore particle in vitro. EMBO J. 15: 2256-2269.

Roy, J., Zheng, B., Rymond, B.C., and Woolford Jr., J.L. 1995. Structurally related but functionally distinct yeast Sm D core small nuclear ribonucleoprotein particle proteins. Mol. Cell. Biol. 15: 445-455.

Rymond, B.C. 1993. Convergent transcripts of the yeast PRP38-SMD1 locus encode two essential splicing factors, including the D1 core polypeptide of small nuclear ribonucleoprotein particles. Proc. Natl. Acad. Sci. 90: 848-852.

Winkler, C., Eggert, C., Gradl, D., Meister, G., Giegerich, M., Wedlich, D., Laggerbauer, B., and Fischer, U. 2005. Reduced $\mathrm{U}$ snRNP assembly causes motor axon degeneration in an animal model for spinal muscular atrophy. Genes \& Dev. 19: 2320 2330.

Zhang, D., Abovich, N., and Rosbash, M. 2001. A biochemical function for the Sm complex. Mol. Cell 7: 319-329. 

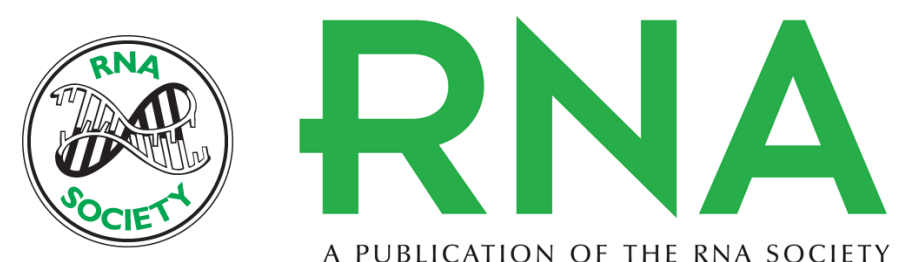

A PUBLICATION OF THE RNA SOCIETY

\section{Sm protein methylation is dispensable for snRNP assembly in Drosophila melanogaster}

Graydon B. Gonsalvez, Kavita Praveen, Amanda J. Hicks, et al.

RNA 2008 14: 878-887

Supplemental http://rnajournal.cshlp.org/content/suppl/2008/03/28/rna.940708.DC1
Material

References This article cites 40 articles, 18 of which can be accessed free at:

http://rnajournal.cshlp.org/content/14/5/878.full.html\#ref-list-1

License

Email Alerting Receive free email alerts when new articles cite this article - sign up in the box at the Service top right corner of the article or click here.

To subscribe to RNA go to:

http://rnajournal.cshlp.org/subscriptions 